ISSN 2075-163X

www.mdpi.com/journal/minerals

Article

\title{
Initial Parallel Arrangement of Extracellular Fibrils Holds a Key for Sheath Frame Construction by Leptothrix sp. Strain OUMS1
}

\author{
Hiromichi Ishihara, Tomoko Suzuki, Hideki Hashimoto, Hitoshi Kunoh and Jun Takada * \\ Department of Material Chemistry, Graduate School of Natural Science and Technology, \\ Okayama University, Okayama 700-8530, Japan; E-Mails: hishihara@cc.okayama-u.ac.jp (H.I.); \\ suzukito@cc.okayama-u.ac.jp (T.S.); hideki-h@cc.okayama-u.ac.jp (H.H.); \\ hkunoh@cc.okayama-u.ac.jp (H.K.)
}

* Author to whom correspondence should be addressed; E-Mail: jtakada@cc.okayama-u.ac.jp; Tel.: +81-86-251-8107; Fax: +81-86-251-8087.

Received: 21 December 2012; in revised form: 18 February 2013 / Accepted: 19 February 2013 / Published: 22 February 2013

\begin{abstract}
Early stages of sheath formation by Leptothrix sp. strain OUMS1 and its derivative sheathless mutant grown in media with or without Fe were examined by light and electron microscopy. Results showed that the initial parallel arrangement of fibrils excreted from the cells holds a key for subsequent construction of the sheath frame and that aqueous-phase Fe interacts with excreted fibrils whether fibrils are parallel-arranged or simply-intermingled.
\end{abstract}

Keywords: Leptothrix sp. OUMS1; sheath formation; bacterial saccharic fibril; deposition of aqueous-phase iron

\section{Introduction}

The genus Leptothrix, a type of Fe/Mn-oxidizing bacteria belonging to the aquatic $\beta$-proteobacteria [1-3], is characterized by the formation of an extracellular, tubular, Fe- or Mn-encrusted sheath, which is closely associated with the Fe/Mn-oxidizing capacities of this genus [4-6]. Although Leptothrix is difficult to isolate [7], four species [8] and seven strains have been distinguished on the basis of phenotypic characteristics and/or 16S rRNA gene sequence [1,6]. Axenic cultures of Leptothrix tend to lose their sheath-forming capacity after repeated transfers $[3,7,9,10]$; 
however, as far as we know, no one has focused on elucidating the cause of this loss during repeated culturing: Is the loss due to a lack of excretion of exopolymers from the bacterial cells, a lack of incorporation of aquatic elements into the exudates or to some other deficiency?

Recently, Suzuki et al. [11] reported that the sheath fibers of L. ochracea had a carbon core of bacterial exopolymers and that aqueous-phase $\mathrm{Fe}$ interacted with $\mathrm{O}$ at the surface of the $\mathrm{C}$ core, resulting in the incorporation of iron oxides at the surface. Furutani et al. [4,5] studied the initial assemblage of bacterial saccharic fibrils and incorporation of elements to form immature sheaths in cultured Leptothrix sp. strain OUMS1 [6] (referred as OUMS1 hereafter) and discovered that basic sheath construction proceeded in two steps: an initial assemblage of bacterial saccharic fibrils from the cell envelope in the direct vicinity of the cells and the subsequent incorporation of aqueous-phase Fe, $\mathrm{P}$, and Si. A detailed comparison of a sheathless mutant with the sheath-forming strain could thus help to understand the mechanisms of sheath formation. Using a spontaneous sheathless mutant that we discovered during repeated transfers of OUMS1 (referred to as SL hereafter), we aimed in the present study to resolve whether SL cells can excrete saccharic materials, whether aquatic inorganics can be incorporated into the materials in the presence or absence of aqueous-phase $\mathrm{Fe}$, and what triggers the initial construction of sheath frame.

\section{Results and Discussion}

On -Fe SGP agar plates, colonies of OUMS1 are normally raised with a rough margin. However, when OUMS1 is subcultured repeatedly (normally 15 or more times), the raised colonies occasionally have a smooth, waterish margin. This change from a rough to smooth margin has often been reported for a mutation in sheath formation of Leptothrix spp. [12-14].

An SL was obtained from a smooth-margined colony, after OUMS1 was subcultured 15 times. When OUMS1 was cultured in +Fe medium, the cells formed chains associated with sheath structures and suspended as a brownish floc, as reported previously [6]. However, in -Fe medium, these chains of living cells were enveloped with a sheath-like film barely distinguishable by light microscopy (Figure 1a) and formed small, whitish flocs. By contrast, SL cells were almost evenly dispersed as single cells or small chains in the medium and did not form any rigid sheaths regardless of $-\mathrm{Fe}$ and + Fe media (Figure 1c). Almost all cells of both OUMS1 and SL were vital in both media (Figure 1b,d).

The possibility that the loss of the sheath-forming capacity of SL might be caused by culture in an artificial media was verified using a method for culturing isolated strains in natural groundwater [15]. A small sample of SL, precultured in -Fe medium for a few days, was injected into a small cellulose dialysis tube bag and then immersed in a container with continuously flowing groundwater for the subsequent culture period. In this groundwater condition, SL never recovered its sheath-forming ability, even after further culturing in $+\mathrm{Fe}$ medium. These results agree with earlier reports on the irreversible loss of sheath formation in spontaneous mutants of L. cholodnii $[10,16]$.

A vaguely delineated, low-contrast material zone, barely distinguishable with light microscopy, appeared to envelop the dispersed, free and the chained SL cells in -Fe medium. In scanning electron microscopy (SEM) observations of the specimens fixed with the glutaraldehyde/OsO $\mathrm{O}_{4}$ mixture, irregularly woven fibrous materials were secreted from SL cells in -Fe medium (Figure 1f, arrow, inset), which corresponded to the extracellular network fibrils of OUMS1 in -Fe medium (Figure 1e, 
inset). Although the microscopical feature of both fibrils look similar, a further verification is required for their qualitative identity.

Figure 1. Differential interference contrast (DIC) (a and c) and fluorescence (b and d) light microscopy and SEM (e and f) images, of Leptothrix sp. strain OUMS1 and sheathless mutant (SL) cells and associated structures cultured in -Fe medium for 3 days. (a) Dense aggregation of chained cells and a few free cells of OUMS1; (b) Green and red fluorescence of these cells after vital staining, green indicating their living state and red indicating dead cells; (c) Scattered, non-aggregated, short chains of a few SL cells; and (d) Their fluorescence after vital staining; (e) OUMS1 cell (BC) and layered fibrils of sheath. Inset: enlarged fibril network; (f) Chains of SL cells and secreted network of fibrils (white arrow). Inset: Enlarged network of loosely woven fibrils. Scale bars, $25 \mu \mathrm{m}$ in (a) $-(\mathbf{d}), 1 \mu \mathrm{m}$ in (e) and (f), and $250 \mathrm{~nm}$ in (e) and (f)-inset.

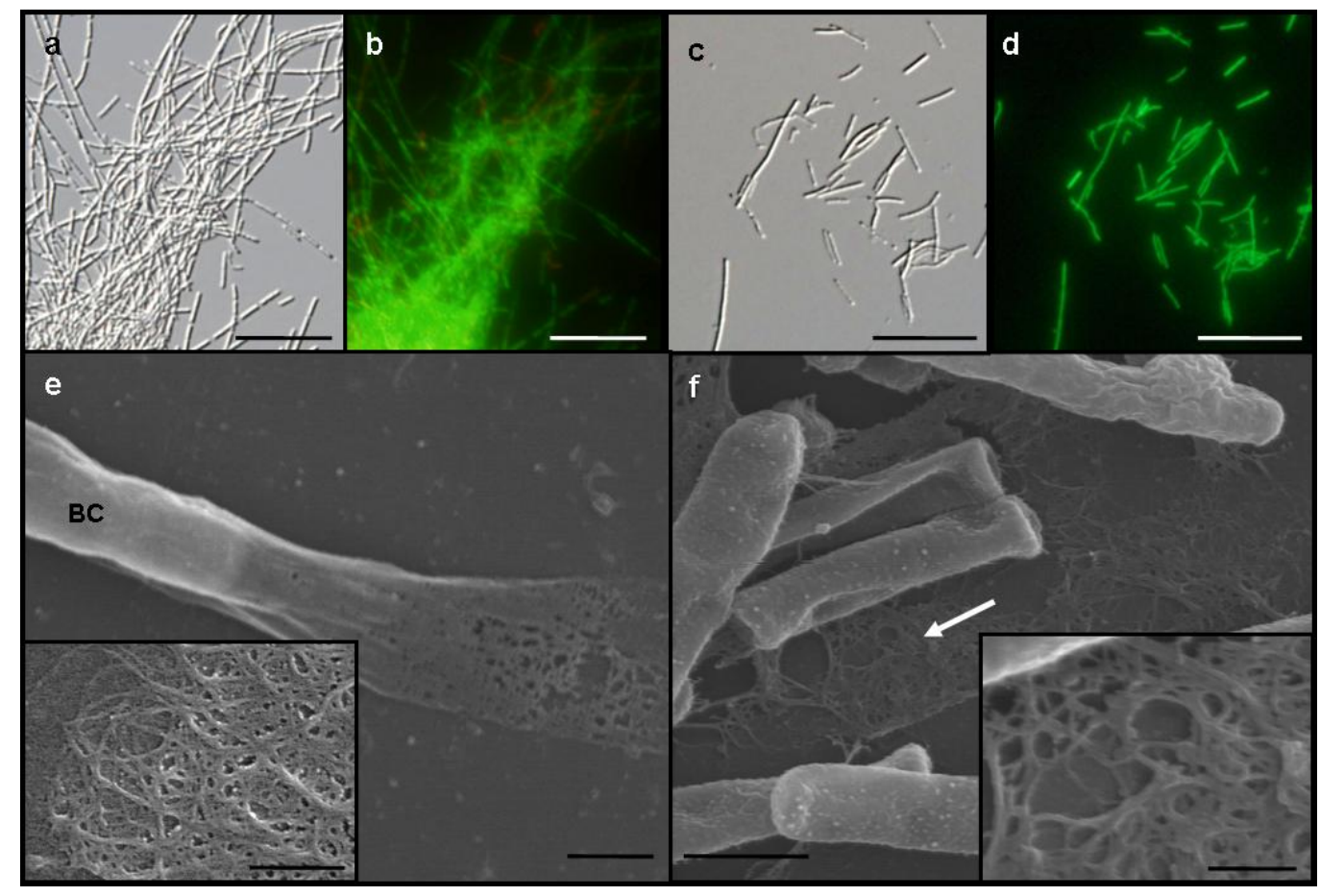

Light microscopy revealed that the majority of free cells and loosely clustered cells of SL were fairly evenly dispersed in -Fe medium after a 3-day incubation (Figure 2a). By contrast, when SL was cultured in $+\mathrm{Fe}$ medium, the majority of the cells aggregated to form ragged clusters without forming any sheath-like structures (Figure 2c). Because sugar-specific fluorescein isothiocyanate (FITC)-conjugated lectins are often used to detect carbohydrates in metal-oxidizing bacteria and their associated structures $[4,17]$, we applied various FITC-conjugated lectins to the SL cells grown in $-\mathrm{Fe}$ or + Fe medium. All test lectins yielded fluorescence from the cells and their associated structures. Of these lectins, Phaseolus vulgaris agglutinin E (PHA-E), which binds specifically to N-acetyl galactosamine, gave the most intense fluorescence, as reported previously in the sheath-forming strain OUMS1 [4]. In -Fe medium fluorescence from the dispersed, free SL cells was barely detectable (Figure 2a,b). Aggregated SL cells and associated structures (Figure 2c,d, dotted arrow) in $+\mathrm{Fe}$ medium, however, fluoresced intensely in contrast to the single or short-chain, free cells (Figure 2c,d, 
solid arrow). Using lectins, Furutani et al. [4] detected saccharic carbohydrates such as glucose, mannose, $\mathrm{N}$-acetyl-galactosamine, and $\mathrm{N}$-acetyl-glucosamine in the immature sheath surrounding OUMS1 chained cells. Their results and the present lectin responses strongly suggest that fibrous materials excreted from SL cells are composed of saccharic carbohydrates. In the present study, we confirmed that all of tested FITC-conjugated lectins pretreated with their respective competitive hapten carbohydrate caused the absence or reduction of fluorescence in the fibril materials and no fluorescence in iron and other unidentified deposits in the medium, suggesting that these lectins specifically bound to their partner saccharic materials. Although the reason that addition of the Fe plates to the medium influences aggregation of SL cells and extracellular polysaccharide secretion are not known, aqueous-phase Fe released from the plates may quantitatively and/or qualitatively modify excretion from the cells. The slight positive fluorescence response around free cells in $-\mathrm{Fe}$ medium is probably due to a low density of saccharic fibrils, which may diffuse into the medium immediately after they are excreted, as was suggested previously [16,18].

Further transmission electron microscopy (TEM) observations of Bi-stained specimens revealed a morphological difference in the electron-dense extracellular materials between OUMS1 and SL cells in $-\mathrm{Fe}$ and $+\mathrm{Fe}$ media. When OUMS1 was cultured in $-\mathrm{Fe}$ medium for 3 days, almost all cells were surrounded by an electron-dense, immature sheath frame (Figure 2e). Enlarged images showed that the bacterial fibrils were almost parallel in the immature sheaths (Figure 2e, inset), similar to the array of the initial inner layer of the fibrous sheath of OUMS1 cultured in $+\mathrm{Fe}$ medium $[4,5]$. By contrast, after 3 days of culture in -Fe medium, dispersed SL cells did not form a sheath-like frame with parallel fibrils (Figure 2f); globular or thread-type projections, which are associated with excretion and transportation of saccharic materials from the cells [4,5], had instead emerged from the surface of the SL cell envelope (Figure 2f, solid arrows). In addition, electron-dense fibrils, scattered in the space near the cells (Figure 2f, dotted arrows), sometimes intermingled but were never parallel-organized (Figure 2f, inset) unlike those excreted from OUMS1. Cultivation of OUMS1 in +Fe medium for 2 days revealed that its cells formed the immature sheath comprised of an inner assembly of parallel-woven fibrils (Figure $2 \mathrm{~g}$, inset, arrows) and an outer, heavily intermingled fibrils with intense electron density (Figure 2g, dotted arrows), as reported by Furutani et al. [4,5]. However, SL cells in + Fe medium even for 3 days failed to construct a well-partitioned sheath frame (Figure $2 \mathrm{~h}$ ). Fibrils were irregularly and loosely intermingled away from the cell in the intervening space (Figure 2h, dotted arrows) and its structure looks very similar with outer fibrils of OUMS1 (Figure 2g, dotted arrow). The inside fibrils were never parallel-arrayed unlike OUMS1 (Figure 2h, inset, arrow). These comparative results gave a strong impression that this first step, parallel arrangement of the excreted fibrils, could hold a role in triggering the subsequent construction of sheath frame.

Furutani et al. [4,5] reported that OUMS1 produced a thin, immature sheath layer comprised of parallel-woven fibrils away from the cell in the intervening space within 1 day of cultivation and that electron-dense, irregularly woven fibrous materials containing metals began to assemble to form the outer layer by day 2. Consistent with their reports, the electron density of outer-layer fibrils of OUMS1 drastically increased after 2 days of growth in $+\mathrm{Fe}$ medium (Figure $2 \mathrm{~g}$, dotted arrows). Amazingly, the electron density was also extremely high in the intermingled fibrils away from the SL cells (Figure $2 \mathrm{~h}$, dotted arrows). 
Figure 2. DIC light (a and $\mathbf{c}$ ) and fluorescence (b and $\mathbf{d}$ ) microscopy of Leptothrix sp. strain OUMS1 or sheathless mutant (SL) cultured in $-\mathrm{Fe}$ or $+\mathrm{Fe}$ medium and treated with FITC-conjugated PHA-E lectin and TEM images of cells and associated structures (e-h). (a) Free, loosely gathered chained cells of SL grown in -Fe medium; and (b) their weakly positive response to the lectin. Scale bar, $20 \mu \mathrm{m}$; (c), (d) Densely aggregated SL cells and associated structures (dotted arrow) and single free or short-chained cells (solid arrow) grown in + Fe medium and their intense fluorescence after lectin treatment. Scale bar, 20 $\mu \mathrm{m}$; (e) OUMS1 cell (BC) in -Fe medium, surrounded by an immature sheath frame of parallel-arranged fibrils across the intervening space (IVS). Inset: enlarged image of parallel-arranged fibrils. Scale bars, $500 \mathrm{~nm}$ and $50 \mathrm{~nm}$ (inset); (f) SL cell in -Fe medium. Thread-like projections are on the cell surface (solid arrows), and excreted fibrils (dotted arrows) are scattered away from the cell. Inset: enlarged image of scattered fibrils. Scale bars, $500 \mathrm{~nm}$ and $50 \mathrm{~nm}$ (inset); (g) OUMS1 cell in +Fe medium, surrounded by a partitioned sheath across the IVS. Sheath was composed of inner layer and outer layer of heavy electron density (dotted arrows). Inset: enlarged image of inner layer (arrows). Scale bars, $250 \mathrm{~nm}$ and $100 \mathrm{~nm}$ (inset); (h) SL cell in +Fe medium, surrounded by heavily aggregated (dotted arrows) but not well-organized fibrils of extremely high electron density. Inset: enlarged image of irregularly clustered fibrils (arrow). Scale bars, $250 \mathrm{~nm}$ and $100 \mathrm{~nm}$ (inset). All images are of 3-day cultures, except for (g) (2-day culture).

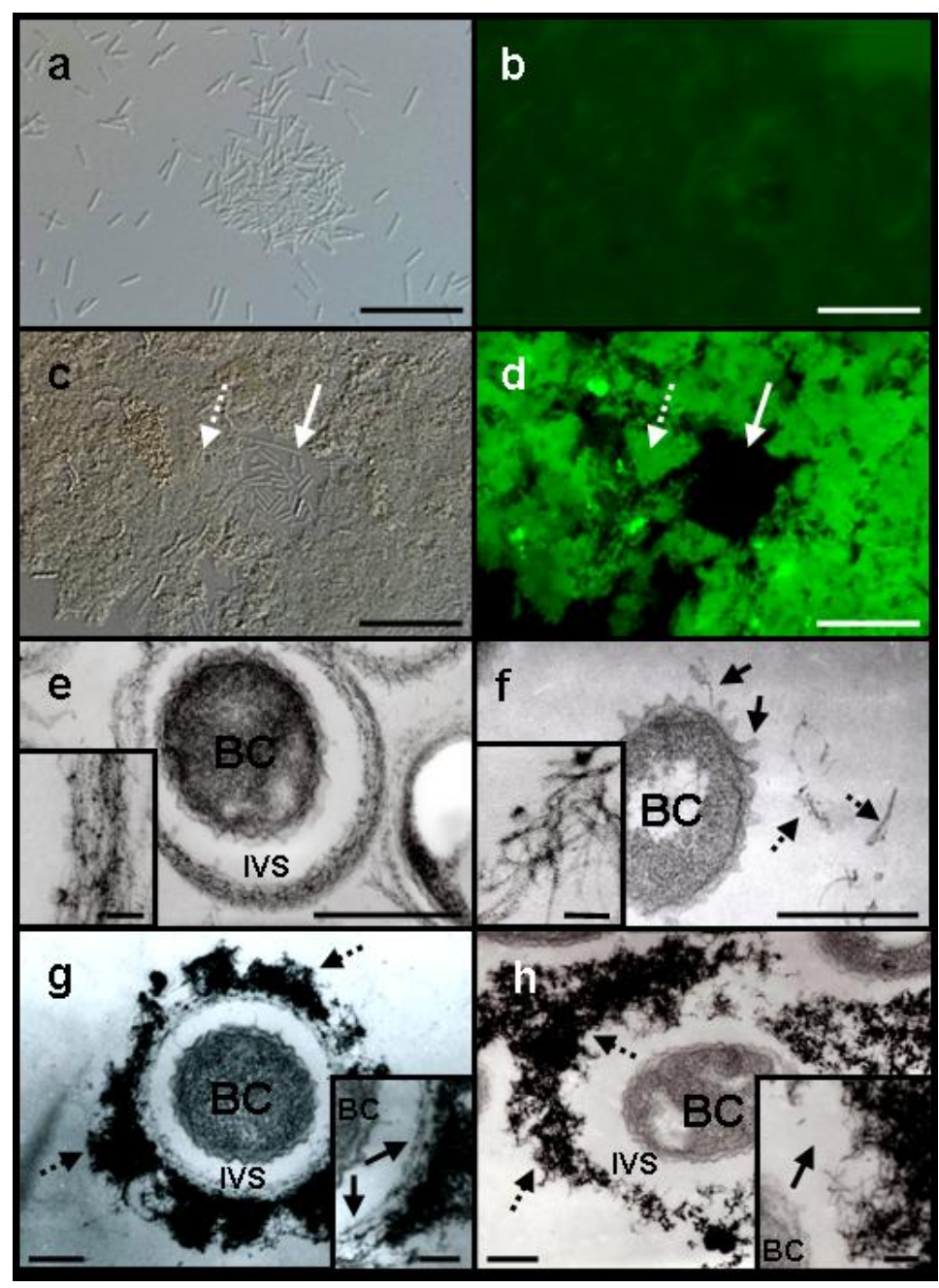


Detailed observation of high-angle annular dark-field scanning transmission electron microscopy (HAADF-STEM) images and energy-dispersive X-ray (EDX) analysis were performed on Bi-stained sections of SL cells from 3-day culture in $+\mathrm{Fe}$ medium to verify whether Fe could be incorporated to excreted saccharic materials. As was expected, Bi staining revealed that the bacterial cell and the fibril assembly around the cell contained the carbohydrate materials (Figure 3a,b). The subsequent EDX analysis detected $\mathrm{Fe}$ only in the fibril assembly but not in the cell (Figure 3a,c), indicating that aqueous-phase Fe obviously interacted with carbohydrate fibrils excreted from SL cells.

Chan et al. [19] hypothesized that organic polymers play important roles in ecosystems by accumulating biologically important elements and that microbial polymers could scavenge iron oxide particles and induce the crystallization of unexpected phases. The present results showing the interaction of the excreted organic fibrils with aqueous-phase Fe, regardless of their forms, apparently support this hypothesis. Through the present experiments, we have concluded that the initial parallel arrangement of bacterial organic fibrils likely holds a key for triggering further construction of the Leptothrix sheath and that the sheathless mutation of SL could be closely related to the loss of a key factor. However, our study raises numerous questions concerning the initial parallel arrangement of excreted fibrils: Are the cells directly or indirectly involved in the arrangement of the fibrils in the space around the cells? If yes, how? Are the organic materials excreted from OUMS1 and SL qualitatively identical? And so on. We are now trying to solve these fundamental queries step by step.

Figure 3. High-angle annular dark-field scanning transmission electron microscopy (HAADF-STEM) images of sheathless mutant (SL) of Leptothrix sp. strain OUMS1 and associated structures in Bi-stained cross sections prepared from 3-day culture in $+\mathrm{Fe}$ medium and elemental maps by energy-dispersive X-ray (EDX) area analysis.

(a) HAADF-STEM image of a cross section of a bacterial cell with the aberrant sheath fibrils; (b) Bi distribution map showing intense signals in both the cells and fibrils; (c) Fe distribution map showing intense signals only in fibrils. Scale bar, $500 \mathrm{~nm}$ in (a)-(c). $\mathrm{BC}$, bacterial cell. IVS, intervening space.

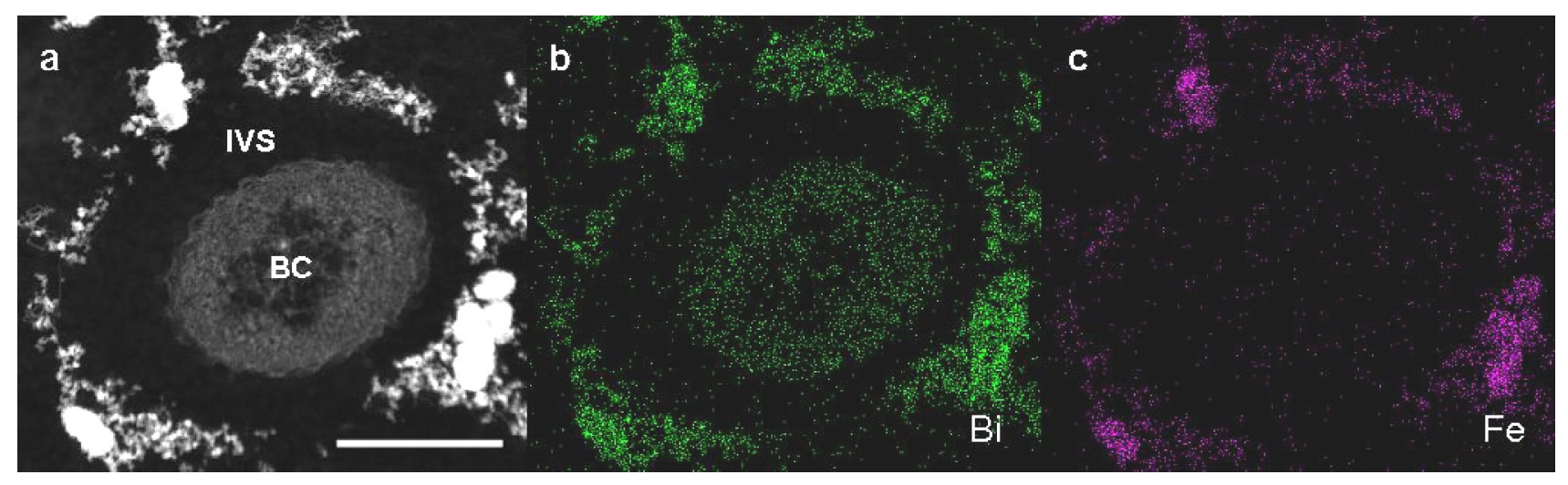

\section{Experimental Section}

\subsection{Sample and Culturing}

Using the protocols of Sawayama et al. [6] and Suzuki et al. [15], we separately cultured OUMS1 (NITE BP-860) and SL in a silicon-glucose-peptone (SGP) liquid medium lacking $\mathrm{FeSO}_{4}$ from 
previously devised SIGP medium [6,15] with or without iron (Fe) plates $\left(10 \times 10 \times 1.2 \mathrm{~mm}^{3} ; 99.9 \%\right.$ purity; Kojundo Chem. Lab., Saitama, Japan) (described as $+\mathrm{Fe}$ and - Fe medium, respectively). Both strains are separately incubated on a rotary shaker at $20^{\circ} \mathrm{C}$ and $70 \mathrm{rpm}$ for 2 or 3 days.

\subsection{Light Microscopy}

The collected cultures were then washed repeatedly with sterilized ultrapure water by centrifugation and were treated with a vital stain to test cell viability using a bacterial viability kit (LIVE/DEAD ${ }^{\circledR}$ Bac Light $^{\mathrm{TM}}$, L7007, Molecular probes, Eugene, OR, USA) or with various FITC-conjugated lectins to test for their hapten sugars by using the protocol of Furutani et al. [4,5], then viewed by differential interference contrast (DIC) and fluorescence microscopy. The tested FITC-conjugated lectins-concanavalin A (Con A), Lens culinaris agglutinin (LCA), peanut (Arachis hypogaea) agglutinin (PNA), PHA-E, and wheat germ agglutinin (WGA) $(10 \mu \mathrm{g} / \mathrm{mL}$ each) — were also confirmed in control assays that pre-treatment of each lectin with the respective competitive hapten carbohydrate caused the absence or reduction of fluorescence in the fibril materials excreted by the bacterial cells, as previously reported [4].

\subsection{Electron Microscopy}

The collected samples were washed repeatedly with sterilized ultrapure water and fixed with a mixture of glutaraldehyde/OsO $\mathrm{O}_{4}$. The fixed samples were dehydrated in a graded ethanol series and freeze dried. The dried samples were platinum $(\mathrm{Pt})$-coated on an aluminum stub and viewed by SEM (S-4300, Hitachi, Tokyo, Japan) [4,5,11]. Alkaline bismuth (Bi)-stained ultrathin sections prepared from glutaraldehyde/OsO${ }_{4}$-fixed specimens were viewed using TEM (H-7500, Hitachi, Tokyo, Japan) [4,5]. Alkaline Bi applied to the thin sections can detect localized carbohydrates [20]. The $\mathrm{Bi}$-stained ultrathin sections on the copper grids were first covered with formvar film using a routine method and then coated with $\mathrm{C}$ to increase the tolerance of the specimen to an intense electron beam. HAADF-STEM and EDX detectors attached to JEOL JEM-2100F (accelerating voltage, $200 \mathrm{kV}$ ) TEM were used for HAADF-STEM imaging and mapping of $\mathrm{Bi}$ and $\mathrm{Fe}$, respectively. Bright field images were also obtained for reference.

\section{Conclusions}

The present study revealed the detailed structure of fibrous materials which were excreted from a spontaneous sheathless mutant SL of Leptothrix sp. strain OUMS1 by comparative observations between OUMS1 and mutant SL, to find the clue of sheath-forming mechanism. Results demonstrated that SL apparently maintained an ability to secrete saccharic fibril materials which interacted with aqueous-phase $\mathrm{Fe}$, but that these materials never formed the well-arranged sheath frame, indicating that the initial parallel arrangement of fibrils which excreted from the cells holds a key for subsequent construction of the sheath frame. 


\section{Acknowledgments}

This study was financially supported by the Special Grant for Education and Research (2008-2013) from the Ministry of Education, Culture, Sports, Science, and Technology, Japan (J.T.), JSPS KAKENHI Grant Number 24780073 (T.S.) and by JST, CREST (2012-2016) (J.T.).

\section{References}

1. Spring, S. The genera Leptothrix and Sphaerotilus. Prokaryotes 2006, 5, 758-777.

2. Takeda, M.; Makita, H.; Ohno, K.; Nakahara, Y.; Koizumi, J. Structural analysis of the sheath of a sheathed bacterium, Leptothrix cholodnii. Int. J. Biol. Macromol. 2005, 37, 92-98.

3. Van Veen, W.L.; Mulder, E.G.; Deinema, M.H. The Sphaerotilus-Leptothrix group of bacteria. Microbiol. Rev. 1978, 42, 329-356.

4. Furutani, M.; Suzuki, T.; Ishihara, H.; Hashimoto, H.; Kunoh, H.; Takada, J. Assemblage of bacterial saccharic microfibrils in sheath skeleton formed by cultured Leptothrix sp. strain OUMS1. J. Mar. Sci. Res. Dev. 2011, S5, doi:10.4172/2155-9910.S5-001.

5. Furutani, M.; Suzuki, T.; Ishihara, H.; Hashimoto, H.; Kunoh, H.; Takada, J. Initial assemblage of bacterial saccharic fibrils and element deposition to form an immature sheath in cultured Leptothrix sp. strain OUMS1. Minerals 2011, 1, 157-166.

6. Sawayama, M.; Suzuki, T.; Hashimoto, H.; Kasai, T.; Furutani, M.; Miyata, N.; Kunoh, H.; Takada, J. Isolation of a Leptothrix strain, OUMS1, from ocherous deposits in groundwater. Curr. Microbiol. 2011, 63, 173-180.

7. Ghiorse, W.C. Biology of iron- and manganese-depositing bacteria. An. Rev. Microbiol. 1984, 38, $515-550$.

8. Emerson, D.; Fleming, E.J.; McBeth, J.M. Iron-oxidizing bacteria: An environmental and genomic perspective. An. Rev. Microbiol. 2010, 64, 561-583.

9. Adams, L.F.; Ghiorse, W.C. Physiology and ultrastructure of Leptothrix discophora SS-1. Arch. Microbial. 1986, 145, 126-135.

10. Emerson, D.; Ghiorse, W.C. Isolation, cultural maintenance, and taxonomy of a sheath-forming strain of Leptothrix discophora and characterization of manganese-oxidizing activity associated with the sheath. Appl. Environ. Microbiol. 1992, 58, 4001-4010.

11. Suzuki, T.; Hashimoto, H.; Ishihara, H.; Kasai, T.; Kunoh, H.; Takada, J. Structural and spatial associations between $\mathrm{Fe}, \mathrm{O}$, and $\mathrm{C}$ in the network structure of the Leptothrix ochracea sheath surface. Appl. Environ. Microbiol. 2011, 77, 7873-7875.

12. Mulder, E.G.; van Veen, WL. Investigations on the Sphaerotilus-Leptothrix Group. Antonie van Leeuwenhoek 1963, 29, 121-153.

13. Rouf, M.A.; Stokes, J.L. Morphology, nutrition and physiology of Sphaerotilus discophorus. Arch. Microbiol. 1964, 49, 132-149.

14. Stokes, J.L.; Powers, M.T. Formation of rough and smooth strains of Sphaerotilus discophorus. Antonie van Leeuwenhoek 1965, 31, 157-164.

15. Suzuki, T.; Ishihara, H.; Furutani, M.; Shiraishi, T.; Kunoh, H.; Takada, J. A novel method for culturing of Leptothrix sp. strain OUMS1 in natural conditions. Minerals 2012, 2, 118-128. 
16. Emerson, D.; Ghiorse, W.C. Ultrastructure and chemical composition of the sheath of Leptothrix discophora SP-6. J. Bacteriol. 1993, 175, 7808-7818.

17. Chan, C.S.; Fakra, S.C.; Emerson, D.; Fleming, E.J.; Edwards, K.J. Lithotrophic iron-oxidizing bacteria produce organic stalks to control mineral growth: implication for biosignature formation. ISME J. 2011, 5, 717-727.

18. Takeda, M.; Kawasaki, Y.; Umezu, T.; Shimura, S.; Hasegawa, M.; Koizumi, J. Patterns of sheath elongation, cell proliferation, and manganese(II) oxidation in Leptothrix cholodnii. Arch. Microbiol. 2012, 194, 667-673.

19. Chan, C.S.; Stasio, G.D.; Welch, S.A.; Girasole, M.; Frazer, B.H.; Nesterova, M.V.; Fakra, S.; Branfield, J.F. Microbial polysaccharides template assembly of nanocrystal fibers. Science 2004, 303, 1656-1658.

20. Park, P.; Ohno, T.; Kato-Kikuchi, H.; Miki, H. Alkaline bismuth stain: A tracer for Golgi vesicles of plant cells. Stain Technol. 1987, 62, 253-256.

(C) 2013 by the authors; licensee MDPI, Basel, Switzerland. This article is an open access article distributed under the terms and conditions of the Creative Commons Attribution license (http://creativecommons.org/licenses/by/3.0/). 